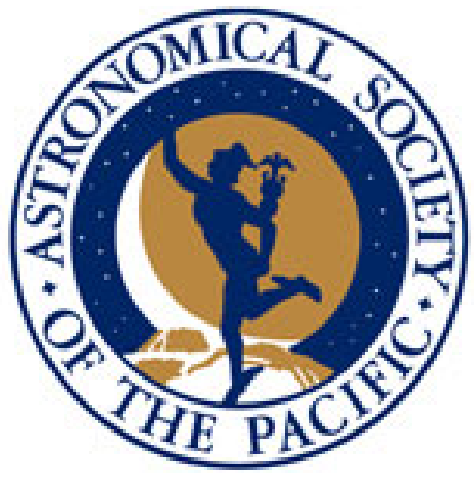

Aperture Photometry Tool Versus SExtractor for Noncrowded Fields

Author(s): Russ R. Laher, Luisa M. Rebull, Varoujan Gorjian, Frank J. Masci, John W. Fowler, Carl Grillmair, Jason Surace, Sean Mattingly, Ed Jackson, Eugean Hacopeans, Nouhad Hamam, Steve Groom, Harry Teplitz, Wei Mi, George Helou, Julian C. van Eyken, Nicholas M. Law, Richard G. Dekany, Gustavo Rahmer, David Hale, Roger Smith, Robert M. Quimby, Eran O. Ofek, Mansi M. Kasliwal, Jeff Zolkower, Viswa Velur, Richard Walters, John Henn ... Reviewed work(s):

Source: Publications of the Astronomical Society of the Pacific, Vol. 124, No. 917 (July 2012), pp. $764-781$

Published by: The University of Chicago Press on behalf of the Astronomical Society of the Pacific

Stable URL: http://www.jstor.org/stable/10.1086/666507

Accessed: 24/09/2012 11:45

Your use of the JSTOR archive indicates your acceptance of the Terms \& Conditions of Use, available at http://www.jstor.org/page/info/about/policies/terms.jsp

JSTOR is a not-for-profit service that helps scholars, researchers, and students discover, use, and build upon a wide range of content in a trusted digital archive. We use information technology and tools to increase productivity and facilitate new forms of scholarship. For more information about JSTOR, please contact support@jstor.org. 


\title{
Aperture Photometry Tool Versus SExtractor for Noncrowded Fields
}

\author{
Russ R. Laher, ${ }^{1}$ Luisa M. Rebull,${ }^{1}$ Varoujan Gorjian, ${ }^{2}$ Frank J. Masci, ${ }^{2}$ John W. Fowler,${ }^{2}$ Carl Grillmair, ${ }^{2}$ \\ Jason Surace, ${ }^{2}$ Sean Mattingly, ${ }^{2}$ Ed Jackson, ${ }^{2}$ Eugean Hacopeans, ${ }^{2}$ Nouhad Hamam, ${ }^{2}$ Steve Groom, ${ }^{2}$ \\ Harry Teplitz, ${ }^{2}$ Wei Mi, ${ }^{2}$ George Helou, ${ }^{2}$ Julian C. van Eyken, ${ }^{3}$ Nicholas M. Law, ${ }^{4}$ Richard G. Dekany, ${ }^{5}$ \\ Gustavo Rahmer, ${ }^{5}$ David Hale, ${ }^{5}$ Roger Smith, ${ }^{5}$ Robert M. Quimby, ${ }^{5}$ Eran O. Ofek,,${ }^{5}$ Mansi M. Kasliwal, ${ }^{5}$ \\ JefF Zolkower, ${ }^{5}$ Viswa Velur, ${ }^{5}$ Richard Walters, ${ }^{5}$ John Henning, ${ }^{5}$ Khahn Bui, ${ }^{5}$ \\ Dan McKenna, ${ }^{5}$ and Shrinivas R. KulKarni ${ }^{5}$ \\ Received 2010 March 29; accepted 2012 April 27; published 2012 June 18
}

\begin{abstract}
Outputs from new software program Aperture Photometry Tool (APT) are compared with similar outputs from SExtractor for sources extracted from $R$-band optical images acquired by the Palomar Transient Factory (PTF), infrared mosaics constructed from Spitzer Space Telescope images, and a processed visible/near-infrared image from the Hubble Legacy Archive (HLA). Two large samples from the PTF images are studied, each containing around $3 \times 10^{3}$ sources from noncrowded fields. The median values of source-intensity relative percentage differences between the two software programs, computed separately for two PTF samples, are $+0.13 \%$ and $+0.17 \%$, with corresponding statistical dispersions of $1.43 \%$ and $1.84 \%$, respectively. For the Spitzer mosaics, a similar large sample of extracted sources for each of channels 1-4 of Spitzer's Infrared Array Camera (IRAC) are analyzed with two different sky annulus sizes, and we find that the median and modal values of source-intensity relative percentage differences between the two software programs are between $-0.5 \%$ and $+2.0 \%$, and the corresponding statistical dispersions range from 1.4 to 6.7\%, depending on the Spitzer IRAC channel and sky annulus. The results for the HLA image are mixed, as might be expected for a moderately crowded field. The comparisons for the three different kinds of images show that there is generally excellent agreement between APT and SExtractor. Differences in source-intensity uncertainty estimates for the PTF images amount to less than $3 \%$ for the PTF sources, and these are potentially caused by SExtractor's omission of the sky background uncertainty term in the formula for source-intensity uncertainty, as well as differing methods of sky background estimation.
\end{abstract}

\section{INTRODUCTION}

In a companion article in this issue of the PASP, Laher et al. (2012) introduced novel interactive software for aperture photometry calculations, called Aperture Photometry Tool (APT), and described the software's major features and capabilities. Because the software is new and heretofore has not been validated in any systematic way, the purpose of the present article is to compare APT results with similar outputs from SExtractor for several large samples of point sources and to statistically assess their mutual agreement. This article treats only relatively isolated or noncrowded sources, which is a first step in quanti-

${ }^{1}$ Spitzer Science Center, California Institute of Technology, Mail Stop 314-6, Pasadena, CA 91125; laher@ipac.caltech.edu.

${ }^{2}$ Infrared Processing and Analysis Center, California Institute of Technology, Mail Stop 100-22, Pasadena, CA 91125.

${ }^{3}$ NASA Exoplanet Science Institute, California Institute of Technology, Mail Stop 100-22, Pasadena, CA 91125.

${ }^{4}$ Dunlap Institute for Astronomy and Astrophysics, University of Toronto, Room 101, Toronto, ON Canada M5S 3H4.

${ }^{5}$ Caltech Optical Observatories, California Institute of Technology, Mail Stop 249-17, Pasadena, CA 91125. tatively validating the software. Aperture photometry has very limited applications in crowded fields, and APT has no special functionality for dealing with such; therefore, this regime is not considered here.

APT is a computer program designed to perform scientific calculations related to aperture photometry, a common datareduction method used in astronomy. The impetus for the advent of this software was the efforts by some coauthors on behalf of NASA to promote math and science in education: specifically, bringing real research into the classroom through a program called NITARP, ${ }^{6}$ the NASA/IPAC Teacher Archive Research Program, and its predecessors (Rebull et al. 2011a). The main feature of the software is that it allows aperture photometry results to be analyzed via a graphical user interface, and this provides a convenient platform for the software's users, both students and astronomy professionals alike, to learn about aperture photometry and gain valuable insights into results particular to their research activities. The first version of the software was released in 2007 November, and many upgrades to the software

\footnotetext{
${ }^{6}$ See http://nitarp.ipac.caltech.edu.
} 
TABLE 1

Properties of PTF IMAGes EMPloyed IN THIS StUdy

\begin{tabular}{ccccccrr}
\hline \hline Case & CCD-exposure moniker $^{\mathrm{a}}$ & Bkg. mode $^{\mathrm{b}}(\mathrm{DN})$ & Bkg. sigma $^{\mathrm{c}}(\mathrm{DN})$ & FWHM $^{\mathrm{d}}(\operatorname{arcsec})$ & Air mass & Pixel scale $^{\mathrm{e}}\left(\operatorname{arcsec}^{2}\right.$ & MZP $^{\mathrm{f}}$ \\
\hline $1 \ldots$ & PTF200912013273_2_o_1997 & 5940 & 1247 & 2.44 & 1.63 & 1.01 \\
$2 \ldots$. & PTF200912015481_2_o_2116 & 3800 & 1308 & 1.92 & 1.18 & 1.01 & 23.707 \\
\hline
\end{tabular}

${ }^{\text {a }}$ Palomar Transient Factory images were acquired on 2009 December 1; both images are from CCDID $=8$.

${ }^{\mathrm{b}}$ Approximate mode of the image histogram.

${ }^{\mathrm{C}}$ Standard deviation of the image histogram.

${ }^{\mathrm{d}}$ Full width at half-maximum of the point-spread function.

${ }^{\mathrm{e}}$ Pixel size on sky.

${ }^{\mathrm{f}}$ Magnitude zero point for conversion of source intensity into apparent magnitude.

have been made since then to augment its capabilities. The software is implemented entirely in the Java programming language and can therefore be installed and used on any computer system that runs a Java Virtual Machine. APT can be downloaded and used free of charge for astronomical-computing purposes. ${ }^{7}$ Laher et al. (2012) give further details about APT.

SExtractor is source-extraction software designed for processing large numbers of images through implementation choices that favor computational speed over high accuracy (Bertin \& Arnouts 1996). Like APT, SExtractor employs the method of aperture photometry, which is generally less accurate than the more sophisticated method of photometry that fits an appropriate point-spread function (PSF) to the image data. Nevertheless, aperture photometry is useful in rough-and-ready applications. We chose SExtractor for this study because it is well established in the astronomical community and, therefore, suitable for use in our comparison with APT.

The next section gives details about the two categories of input images that we highlight in this article and that were fed into the two software programs (APT and SExtractor) to generate the results for our analysis. Section 3 explains the method followed in the software-output comparison. Section 4 presents the comparison results and discusses the findings. The conclusion reached is given in the last section, along with future work that may be fruitfully pursued.

\section{INPUT DATA}

The input data described in this section were chosen for convenience and to illustrate various aspects of APT versus SExtractor performance. Although input data from three telescopes and instruments are considered herein, testing input data from other projects is beyond the scale of this article. APT can read most kinds of astronomical images in FITS format, including those with multiple data planes and extensions. ${ }^{8}$ Consequently, APT also functions as a handy FITS viewer.

\footnotetext{
${ }^{7}$ See http://www.aperturephotometry.org.

${ }^{8}$ See http://fits.gsfc.nasa.gov.
}

\subsection{Palomar Transient Factory Images}

As listed in Table 1 and shown in Figure 1, our study includes two different CCD-array images from the Palomar Transient Factory (Law et al. 2009; Rau et al. 2009): PTF200912013273_ 2_o_1997 (case 1) and PTF200912015481_2_o_2116 (case 2). The PTF camera has 11 operational CCD arrays, and segregated PTF CCD-array images have $2048 \times 4096$ pixels, where a pixel on a side is 1.01 " across the sky. Both images are $60 \mathrm{~s}$ exposures from the night of 2009 December 1 and from the same CCD array $(C C D I D=8)$. This CCD array, at the time the images were
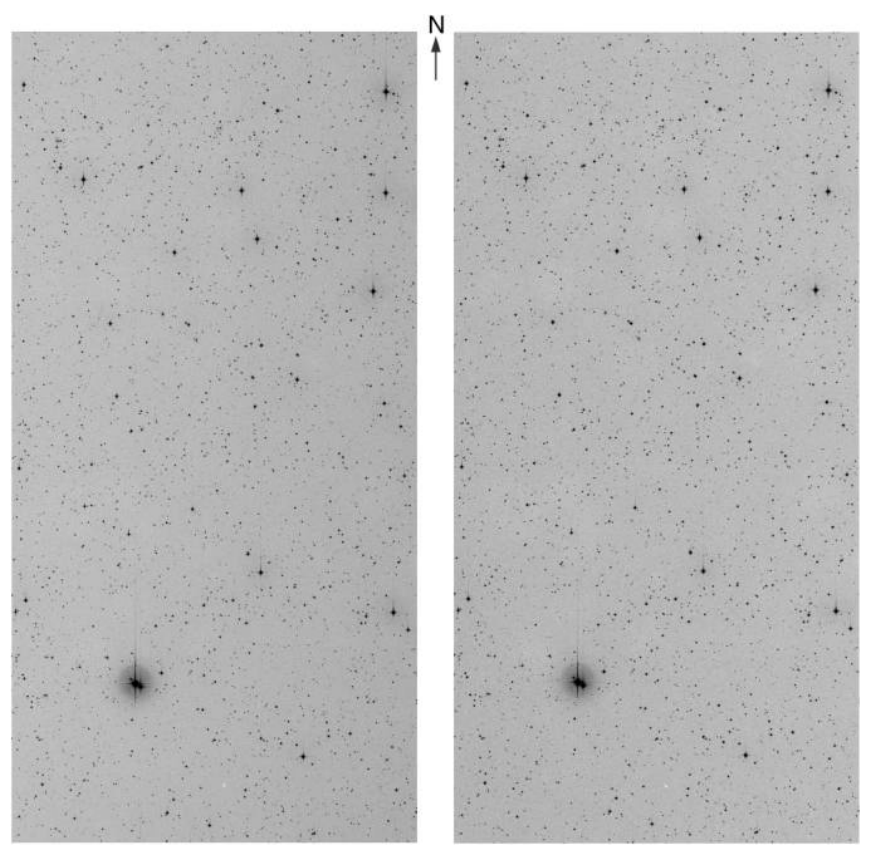

FIG. 1.—CCDID=8 images PTF200912013273_2_o_1997 (left; case 1) and PTF200912015481_2_o_2116 (right; case 2) from the Palomar Transient Factory. These are $60 \mathrm{~s}$ exposures acquired $\approx 5 \mathrm{hr}$ apart with the $R$ filter, at approximately the same sky position of R.A. $=08^{\mathrm{h}} 15^{\mathrm{m}} 6^{\mathrm{s}}$ and decl. $=+11^{\circ} 46^{\prime} 34^{\prime \prime}$. The sky extent is $\approx 69^{\prime}$ along the vertical dimension of each image. These representations were made using the mJPEG module of NASA/IPAC Infrared Science Archive's Montage software, with linear stretches separately applied over different limited data ranges for the two images and inverted grayscale, in order to enhance both the contrast and fainter image features. 
taken, had a gain of $1.4 e^{-} \mathrm{DN}^{-1}$ (where $\mathrm{DN}$ stands for data number), read noise of $5.9 e^{-}$, and dark current of less than $0.1 e^{-} \mathrm{s}^{-1}$. The saturation limit of each CCD-array pixel is $\approx 51,000 \mathrm{DN}$ ( or $7.14 \times 10^{4} e^{-}$). The two images were acquired within about $5 \mathrm{hr}$ of each other, with the $R$ filter, and field-ofview center at approximately the same sky position of R.A. = $08^{\mathrm{h}} 15^{\mathrm{m}} 6^{\mathrm{s}}$ and decl. $=+11^{\circ} 46^{\prime} 34^{\prime \prime} \quad($ PTFFIELD $=3256)$. Because the two images were taken at different times during the night, they have differing global sky background levels, which can be used to assess the effect of sky background subtraction on the resulting source intensities. Also, the two images have slightly different PSFs (from seeing variations) and image-pixel alignments on the sky (due to expected slight telescope mispointings from exposure to exposure on the same target). Prior to the analysis reported herein, the raw PTF images were processed with IPAC-PTF data-reduction pipelines (Grillmair et al. 2010), which performed the bias subtraction, flat-field correction, and astrometric and photometric calibrations. The processed-image histogram for case 1 peaks at about $5940 \mathrm{DN}$, and for case 2 at $3800 \mathrm{DN}$.

\subsection{Spitzer IRAC Mosaics}

We also consider the aperture photometry of sources in mosaics of images acquired by the Spitzer Space Telescope (Werner et al. 2004). The raw images used to generate the mosaics were taken in the vicinity of Cometary Globule 4 (CG4) at R.A. $=07^{\mathrm{h}} 34^{\mathrm{m}} 09^{\mathrm{s}}$, decl. $=-46^{\circ} 54.3^{\prime}$ by Spitzer's Infrared Array Camera (IRAC) in four infrared passbands:
3.6, 4.5, 5.8, and $8.0 \mu \mathrm{m}$ (Fazio et al. 2004). The passbands correspond to IRAC instrument channels $1-4$, respectively. We downloaded the associated processed individual images from the Spitzer Heritage Archive for AORKEY 24250880 and employed MOPEX (Makovoz and Marleau 2005) to clean up instrument artifacts, resample the processed images, and create mosaics for the four infrared channels (see Fig. 2). These data were analyzed in a recent study to identify young stellar objects (Rebull et al. 2011b). The mosaics were computed to have a pixel scale of $0.610^{\prime \prime}$ per pixel (which is about half of the native IRAC pixel scale) and a size of $6515 \times 5293$ pixels. Only around half of each mosaic is filled with actual data, roughly an area of $50 \times 30 \mathrm{arcmin}^{2}$, because of nonalignment of the sky coverage with the mosaic reference frame (the pixels without data contain NaN [not a number] values). The backgrounds of the mosaics include a substantial amount of nebulosity, which increases in intensity with longer infrared wavelengths. The physical units of the image data are $\mathrm{MJy} \mathrm{sr}^{-1}$. The number of images co-added to generate the mosaics at a given pixel location, or depth of coverage, varies across the imaged area of the mosaics and is as deep as 15 images, with typical (bimodal) values of three and eight images. Table 2 gives additional details about the Spitzer IRAC mosaics.

\subsection{Hubble Space Telescope Image}

Although this article focuses on software performance for noncrowded fields, we chose to include a moderately crowded field taken by the Hubble Space Telescope (HST), in order to

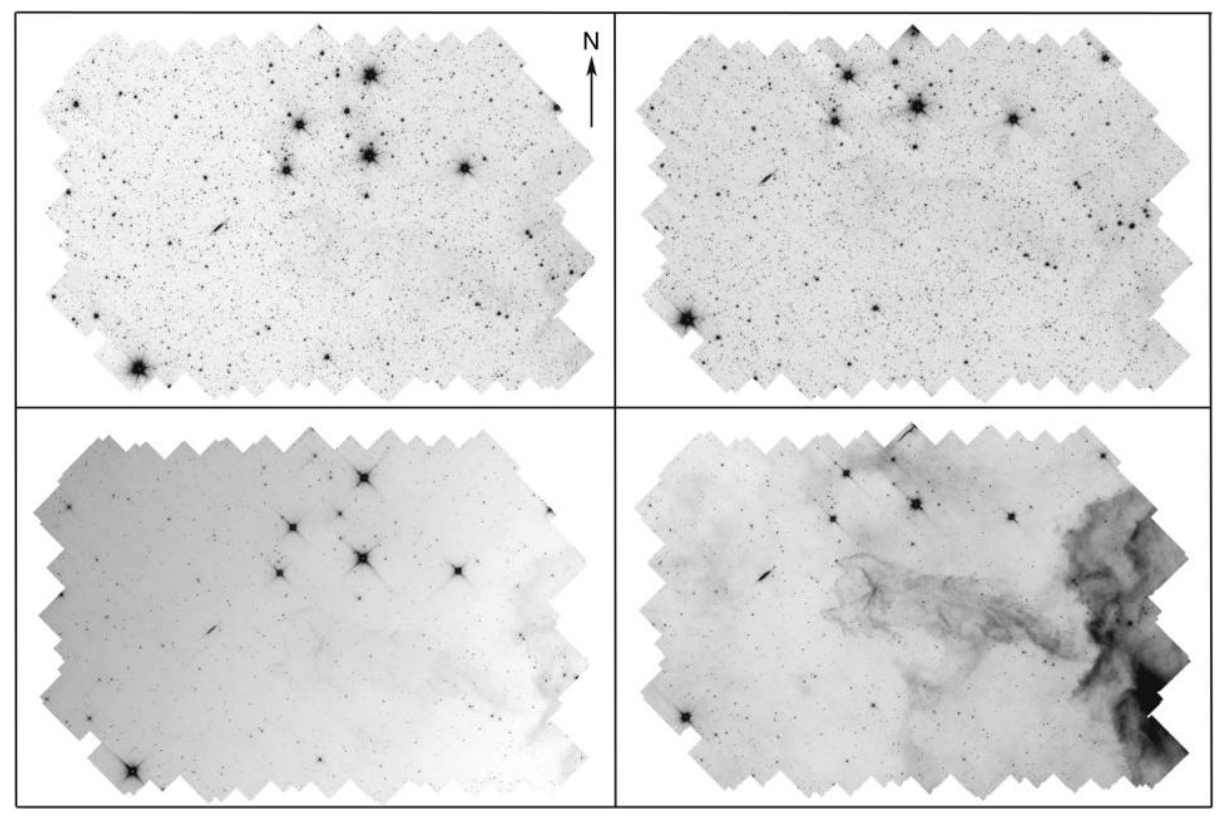

FIG. 2.-Spitzer IRAC mosaics for channels 1 and 2 (top) and channels 3 and 4 (bottom). The sky area shown in each mosaic is roughly $50 \times 30$ arcmin ${ }^{2}$. The equatorial coordinates of the center of the sky area shown are approximately R.A. $=07^{\mathrm{h}} 33^{\mathrm{m}} 53^{\mathrm{s}}$ and decl. $=-46^{\circ} 57^{\prime} 08^{\prime \prime}$. These representations were made using the mJPEG module of NASA/IPAC Infrared Science Archive's Montage software, with linear stretches separately applied over different limited data ranges for the four images and inverted grayscale, in order to enhance both the contrast and fainter image features. 
TABLE 2

Properties of SPITZER IRAC Mosaics EMPloyed in this Study

\begin{tabular}{|c|c|c|c|c|c|c|}
\hline Channel $^{\mathrm{a}}$ & $\begin{array}{l}\text { Bkg. mode }{ }^{\mathrm{b}} \\
\left(\mathrm{MJy} \mathrm{sr}^{-1}\right)\end{array}$ & $\begin{array}{l}\text { Bkg. } \operatorname{sigma}^{\mathrm{c}} \\
\left(\mathrm{MJy} \mathrm{sr}^{-1}\right)\end{array}$ & $\begin{array}{l}\text { FWHM }^{\mathrm{d}} \\
(\operatorname{arcsec})\end{array}$ & $\begin{array}{l}\text { Pixel scale } \\
\quad(\operatorname{arcsec})\end{array}$ & Aper. corr. ${ }^{\mathrm{f}}$ & $\mathrm{MZP}^{\mathrm{g}}$ \\
\hline 1 & 0.036 & 3.06 & 1.7 & 0.610 & 1.124 & 18.77 \\
\hline$\ldots \ldots \ldots$ & 0.063 & 1.99 & 1.7 & 0.610 & 1.127 & 18.28 \\
\hline 3 & 1.09 & 6.43 & 2.4 & 0.610 & 1.143 & 17.80 \\
\hline$\ldots \ldots \ldots$ & 1.5 & 3.64 & 2.4 & 0.610 & 1.234 & 17.16 \\
\hline \multicolumn{7}{|c|}{$\begin{array}{l}{ }^{a} \text { Mosaics made using MOPEX software. IRAC instrument channels } 1-4 \text { correspond to } 3.6,4.5,5.8 \text {, and } \\
.0 \mu \text { m infrared passbands, respectively. }\end{array}$} \\
\hline \multirow{2}{*}{\multicolumn{7}{|c|}{$\begin{array}{l}\text { 'Approximate mode of the mosaic's histogram. The histogram for channel } 3 \text { is trimodal, and the middle mode } \\
\text { is listed. }\end{array}$}} \\
\hline & & & & & & \\
\hline \multicolumn{7}{|c|}{${ }^{\mathrm{c}}$ Standard deviation of the mosaic's histogram. } \\
\hline \multicolumn{7}{|c|}{${ }^{\mathrm{d}}$ Full width at half-maximum of the point-spread function. } \\
\hline \multicolumn{7}{|c|}{${ }^{\mathrm{e}}$ Mosaic pixel size on sky. } \\
\hline \multicolumn{7}{|c|}{${ }^{\mathrm{f}}$ Aperture correction, from the Spitzer IRAC instrument handbook. } \\
\hline
\end{tabular}

assess the impact of veering slightly outside of the noncrowded regime. We downloaded visible/near-infrared HST drizzle image HST_10048_a1_ACS_HRC_F814W from the Hubble Legacy Archive (HLA). The image is shown in Figure 3. These data have been previously used in a study to detect new variable stars (Fiorentino et al. 2008). The $1076 \mathrm{~s}$ exposure of NGC 121 was generated from dithered image data acquired on 2005 June 19 by the Advanced Camera for Surveys/High Resolution Channel (ACS/HRC) detector with the F814W filter.

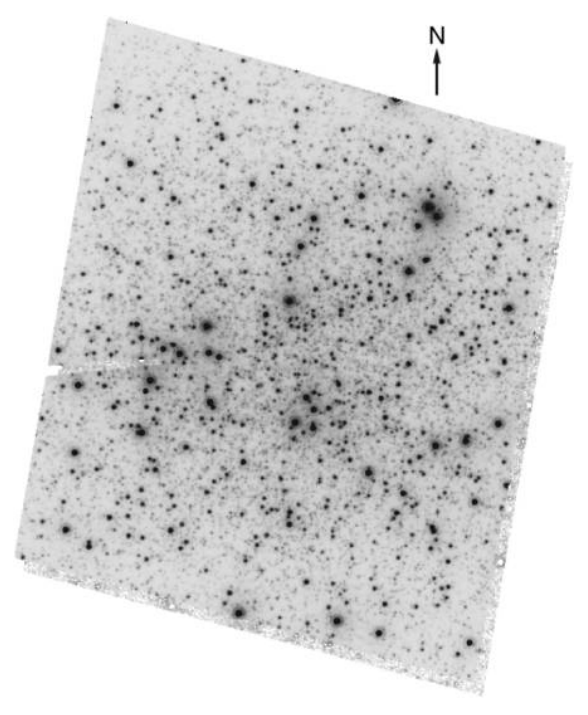

FIG. 3._Hubble Space Telescope drizzle image HST_10048_a1_ACS_ HRC_F814W from the Hubble Legacy Archive. This is a 1076 s exposure acquired with the F814W filter, centered on a sky position of approximately R.A. $=00^{\mathrm{h}} 26^{\mathrm{m}} 49^{\mathrm{s}}$ and decl. $=-71^{\circ} 32^{\prime} 07^{\prime \prime}$. The sky extent is $\approx 40^{\prime \prime}$ along the vertical dimension of the image. This representation was made via screen capture of APT's thumbnail image, shown with a logarithmic stretch and inverted grayscale, in order to enhance both the contrast and fainter image features.
The observations are centered on a sky position of approximately R.A. $=00^{\mathrm{h}} 26^{\mathrm{m}} 49^{\mathrm{s}}$ and decl. $=-71^{\circ} 32^{\prime} 07^{\prime \prime}$. The sky extent of the drizzle image is $\approx 40^{\prime \prime}$ along the vertical dimension. The HLA product had its background of $32.63 e^{-} \mathrm{s}^{-1}$ subtracted as part of the standard HLA multidrizzle processing (Koekemoer et al. 2002). Cosmic rays and geometric distortion are also removed in the standard processing. The image has $1500 \times 1500$ pixels, where a pixel on a side is $0.025^{\prime \prime}$ across the sky.

\section{METHOD}

\subsection{General}

We assessed the calculational results of Aperture Photometry Tool, version 1.0.5, by comparing its outputs with similar results from SExtractor, version 2.8.6, a recent version. That is, for a large number of source detections extracted from the image of interest, we compared the aperture photometry results from the two software programs for a fixed circular aperture on a sourceby-source basis.

The relative percentage difference between the SExtractor result and the APT result for source $i$ is defined here as

$$
\Delta_{\mathrm{rel}}^{i}=100 \times \frac{f_{\mathrm{SEx}}^{i}-f_{\mathrm{APT}}^{i}}{f_{\mathrm{SEx}}^{i}},
$$

where $f_{\mathrm{SEx}}^{i}$ is the source intensity from SExtractor and $f_{\mathrm{APT}}^{i}$ is the source intensity from APT. We used this figure of merit to compare source intensities from SExtractor versus APT, with SExtractor's results defined as the standard for present purposes. We also used similar equations to compare sky backgrounds and source-intensity uncertainties from the two software programs.

The calculation of source intensity involves integrating the measured counts from pixels and subpixels within the aperture and subtracting the sky background contribution computed 
from a sky annulus around the aperture. We configured SExtractor to compute the local background, (i.e., where BACKРНOTO TYPE=LOCAL), as is usual for APT. A notable difference between the local-background calculational methods used by the two software programs is that SExtractor uses a square sky annulus, while APT's is circular. SExtractor's input parameters BACK_SIZE and BACKPHOTO_THICK control the outer dimension and thickness of the sky annulus, respectively. These are integer parameters, as are APT's inner and outer sky annulus radii. For a given input image, we took care to ensure that the size of the sky annulus of the APT calculation was similar to that of the SExtractor calculation.

\subsection{Specifics for PTF Images}

For the PTF images, we used an aperture radius of 5 pixels (or 5.05") in the aperture photometry calculations, because this is the point at which randomly sampled curve-of-growth plots start to level off. A larger or smaller aperture radius also would have been acceptable for the purposes of this comparison. For the sky annulus sizing in the local-background calculations, we set BACK_SIZE=80 and BACKPHOTO_THICK=34 pixels for SExtractor, and $r_{\text {in }}=6$ and $r_{\text {out }}=40$ pixels for APT.

SExtractor source-list generation yielded 4102 sources for case 1 and 4945 sources for case 2 (see Table 3 ). We then filtered the SExtractor results for sources with moderate stellarity (SExtractor CLASS_STAR>0 .5), peak pixel value greater than 4 times the root-mean-squared sky background to exclude noise-confused sources, and high degree of "cleanliness." The latter translates to having SExtractor FLAGS $=0$ for all sources. According to the software documentation, this means that the source has no neighbors bright and close enough to significantly bias the photometry, has no significant number of bad pixels, is not blended with other sources, has no pixels that are saturated or close to saturation, is not near an image boundary, and has complete and uncorrupted data; in addition, its photometry can be computed without memory overflow (Holwerda 2005). We also verified that each filtered source has no neighboring sources within $5^{\prime \prime}$. The filtering is to ensure that no crowded sources are compared, so that crowded sources are excluded from our comparisons. Note that the effect of thresholding on stellarity becomes random for very dim sources. Table 3 lists the numbers of sources that remained after the filtering for the two cases: 2727 sources for case 1, and 3170 sources for case 2 .

Next, we created APT source lists of equatorial coordinates for the two cases using the filtered source lists, which we subsequently processed using APT's source-list tool. When setting up APT for the processing, we inputted the aforementioned radii for the aperture and sky annulus, selected the decimal degree representation of equatorial coordinates from the Preferences menu, and selected the median sky subtraction model B from the More Settings panel. We also set 0.1 pixels for the computational step size. Otherwise, default APT settings were used.

The APT calculations for the number of filtered sources involved in the PTF cases took 30-40 minutes per case on a Mac laptop with $2.53 \mathrm{GHz}$ Intel Core 2 Duo processor, 4 GBytes of memory, and running Mac OS X version 10.5.8 (each source completed in $\approx 700 \mathrm{~ms}$ ). By comparison, SExtractor took $\approx 3.2 \mathrm{~ms}$ per source. The slow computing time resulted, in part, because the APT calculations were done with 0.1 subpixel resolution, and it can be speeded up by approximately a factor of 2 if 1.0 pixel resolution is selected. The primary reason for the huge difference in computational speed between APT and SExtractor is that the former is implemented in Java and the latter is implemented in the $\mathrm{C}$ programming language. Processing speed is not a primary concern for APT, however, because it was designed for visualizing aperture photometry calculations for relatively small numbers of sources. Nevertheless, this article demonstrates that APT can be practically employed to analyze thousands of sources and at a faster rate by increasing the calculational step size. Despite the slower speed, APT's

TABLE 3

Results for Palomar Transient Factory Cases

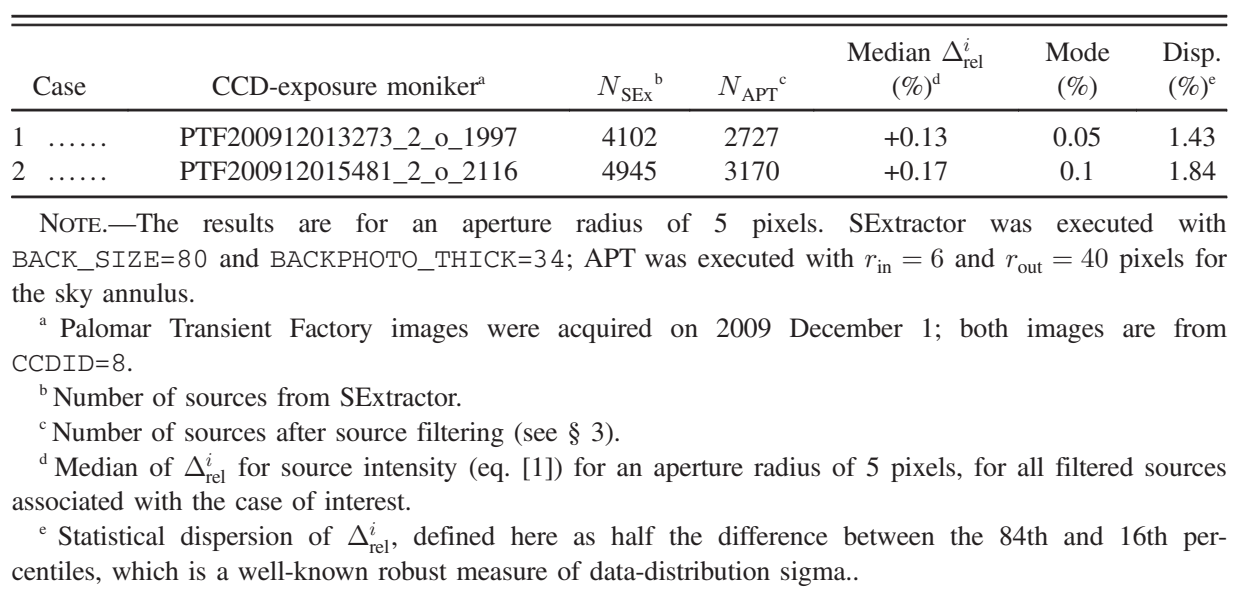



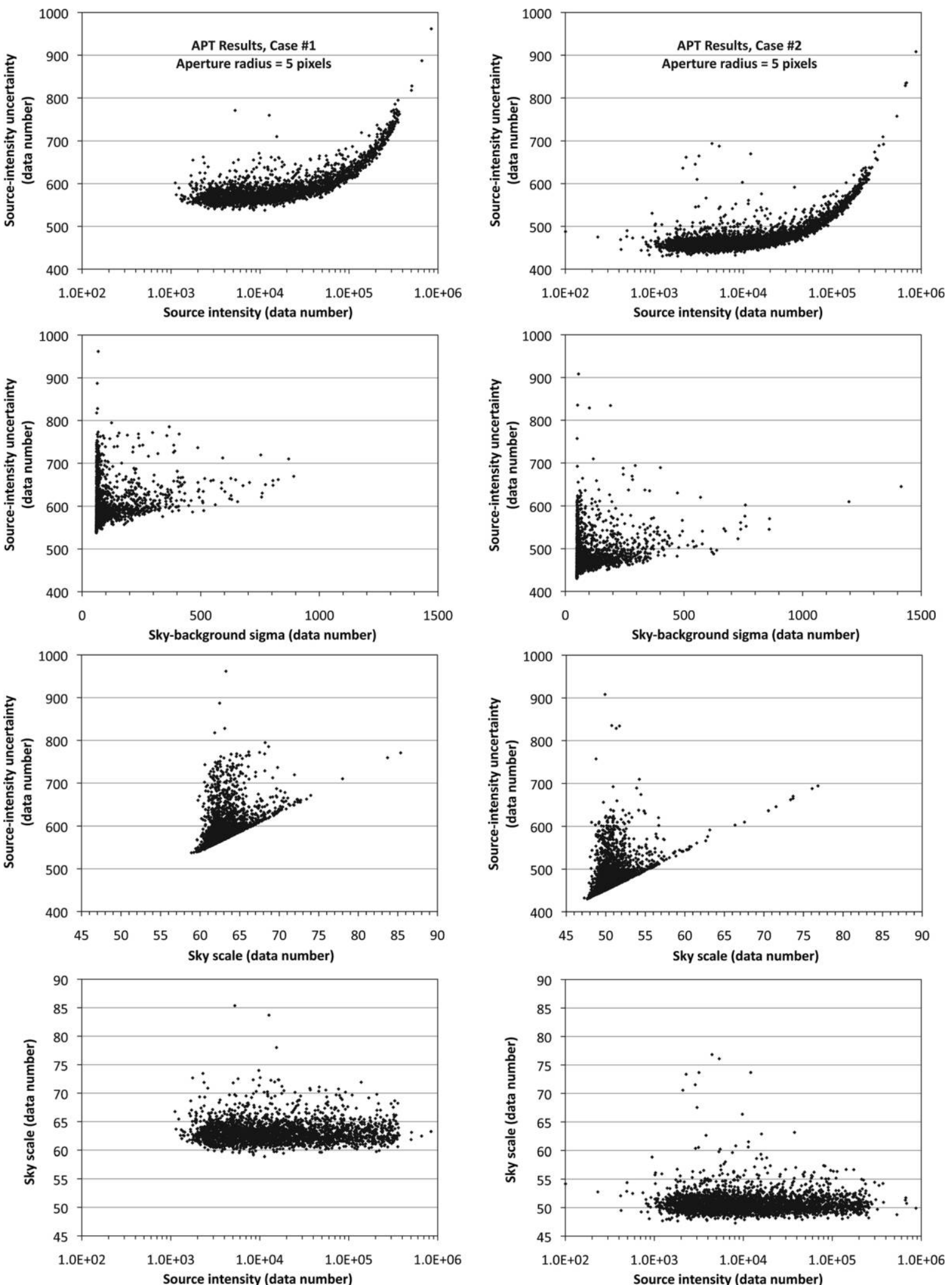

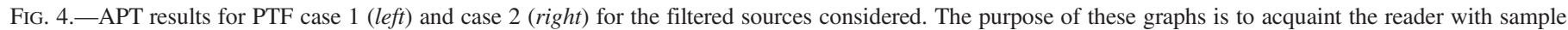

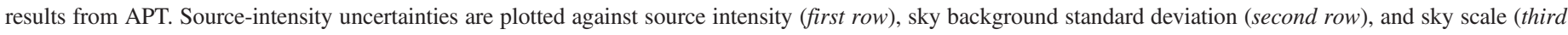

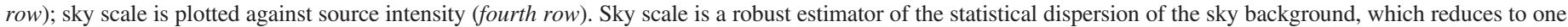
standard deviation in the special case of a Gaussian distribution. 
TABLE 4

RESUlTS FOR SPITZER IRAC MOSAIC CASES

\begin{tabular}{|c|c|c|c|c|c|c|c|}
\hline Case & Channel $^{\mathrm{a}}$ & $\begin{array}{l}\text { Sky annulus }^{\mathrm{b}} \\
\text { (pixels) }\end{array}$ & $N_{\mathrm{SEx}}{ }^{\mathrm{c}}$ & $N_{\text {APT }}{ }^{\mathrm{d}}$ & $\begin{array}{l}\text { Median } \Delta_{\text {rel }}^{i} \\
(\%)^{\mathrm{e}}\end{array}$ & $\begin{array}{l}\text { Mode } \\
(\%)\end{array}$ & $\begin{array}{l}\operatorname{Disp}^{f} \\
(\%)\end{array}$ \\
\hline A1 & 1 & 80,$34 ; 6,40$ & 22642 & 7853 & +2.00 & +0.3 & 4.28 \\
\hline$\ldots \ldots$ & 2 & 80,$34 ; 6,40$ & 15781 & 6700 & +1.20 & +0.2 & 3.27 \\
\hline$\ldots \ldots \ldots$ & 3 & 80,$34 ; 6,40$ & 5670 & 2108 & +0.26 & +0.2 & 1.44 \\
\hline$\ldots \ldots$ & 4 & 80,$34 ; 6,40$ & 4603 & 1561 & +0.20 & +0.3 & 2.85 \\
\hline$\ldots \ldots \ldots$ & 1 & 28,$8 ; 6,14$ & 21892 & 8405 & +0.92 & +1.2 & 5.59 \\
\hline ....... & 2 & 28,$8 ; 6,14$ & 13524 & 6818 & +0.38 & +0.9 & 5.54 \\
\hline$\ldots \ldots \ldots$ & 3 & 28,$8 ; 6,14$ & 3638 & 1991 & +0.09 & +1.4 & 3.73 \\
\hline$\ldots \ldots \ldots$ & 4 & 28,$8 ; 6,14$ & 3132 & 1557 & -0.47 & +0.7 & 6.70 \\
\hline
\end{tabular}

NOTE.-The results are for an aperture radius of 6 pixels (or $3.66^{\prime \prime}$ ). Cases $\mathrm{A} i$ and $\mathrm{B} i$, where $i=1, \ldots, 4$ is the infrared channel number, correspond to the same Spitzer IRAC mosaics processed with two different sky annulus sizes: large (cases $\mathrm{A} i$ ) and small (cases $\mathrm{B} i$ ).

${ }^{a}$ Spitzer IRAC mosaics of CG4 made using MOPEX software. Channels 1-4 correspond to infrared passbands centered at 3.6, 4.5, 5.8, and $8.0 \mu \mathrm{m}$.

${ }^{\mathrm{b}}$ SExtractor input parameters BACK_SIZE and BACKPHOTO_THICK specify sky annulus outer dimension and thickness; APT inner and outer sky annulus radii.

${ }^{\mathrm{c}}$ Number of sources from SExtractor.

${ }^{\mathrm{d}}$ Number of sources after filtering (see $\S 3$ ).

${ }^{\mathrm{e}}$ Median of $\Delta_{\text {rel }}^{i}$ for source intensity (eq. [1]) computed with an aperture radius of 6 pixels, for all filtered sources associated with the case of interest.

${ }^{\mathrm{f}}$ Statistical dispersion of $\Delta_{\text {rel }}^{i}$ for source intensity, defined here as half the difference between the 84th and 16 th percentiles, which is a well-known robust measure of data-distribution sigma.

interactive capabilities facilitate analysis insight that is not readily available through SExtractor.

The plots in Figure 4 show APT results computed for cases 1 (left) and 2 (right). The top panels illustrate the relationship between source intensity and source-intensity uncertainty, where each data point represents one of the corresponding image's filtered sources. The lower source-intensity cutoff is different for the two cases because of the differing sky background levels of the images. The second row of panels in Figure 4 plot source-intensity uncertainty versus sky background sigma. As defined in Laher et al. (2012), the sky background sigma is the standard deviation of samples in the sky annulus after the sky outliers have been rejected (as of version 1.0.5, APT does 3- $\sigma$ outlier rejection prior to computing the sky background statistics). The third row of panels plot sourceintensity uncertainty versus sky scale, which is a robust estimator of sky background statistical dispersion that reduces to one standard deviation in the special case of a Gaussian distribution. The source-intensity uncertainty, sky background sigma, and sky scale are more fully defined in Laher et al. (2012). The bottom panels in Figure 4 plot sky scale versus source intensity and remind us that the sources with large sky scale are not necessarily the brightest.

\subsection{Specifics for Spitzer IRAC Mosaics}

The aperture radius was set to 6 pixels (or 3.66") for the aperture photometry calculations that we performed on the Spitzer IRAC mosaics (note that this is a different aperture radius from that used for the PTF images introduced in the previous subsection). The driver for this choice is that this aperture size is one recommended by the Spitzer IRAC instrument support team to be optimal. ${ }^{9}$ An aperture this size captures roughly $75-90 \%$ of the flux density from a given source, and so a channel-dependent aperture correction is normally needed to yield accurate source intensities (see Table 2). The aperture correction also depends on the geometry and sizing of the aperture. For our purposes, since we are comparing APT versus SExtractor results, we can ignore the aperture correction (at least for the basic comparison), but we nevertheless deemed it desirable to employ a reasonable aperture size in our work here.

We ran APT and SExtractor for two different sky annuli on the four mosaics, resulting in cases $\mathrm{A} i$ and $\mathrm{B} i$, where $i=1, \ldots, 4$ is the infrared channel number. For cases $\mathrm{A}_{i}$, we set BACK_SIZE $=80$ and BACKPHOTO_THICK=34 pixels for SExtractor and $r_{\text {in }}=6$ and $r_{\text {out }}=40$ pixels for APT. For cases $\mathrm{B} i$, we set BACK_SIZE $=28$ and BACKPHOTO_THICK= 8 pixels for SExtractor and $r_{\text {in }}=6$ and $r_{\text {out }}=14$ pixels for APT. (We changed BACK_SIZE because we believe this controls the width of SExtractor's square annulus, although the documentation is admittedly unclear on this point.) These two basic cases afford us the opportunity of comparing APT versus SExtractor results for large and small sky annulus sizes.

\footnotetext{
${ }^{9}$ See the IRAC Instrument Handbook: http://irsa.ipac.caltech.edu/data/ SPITZER/docs/irac/iracinstrumenthandbook/.
} 

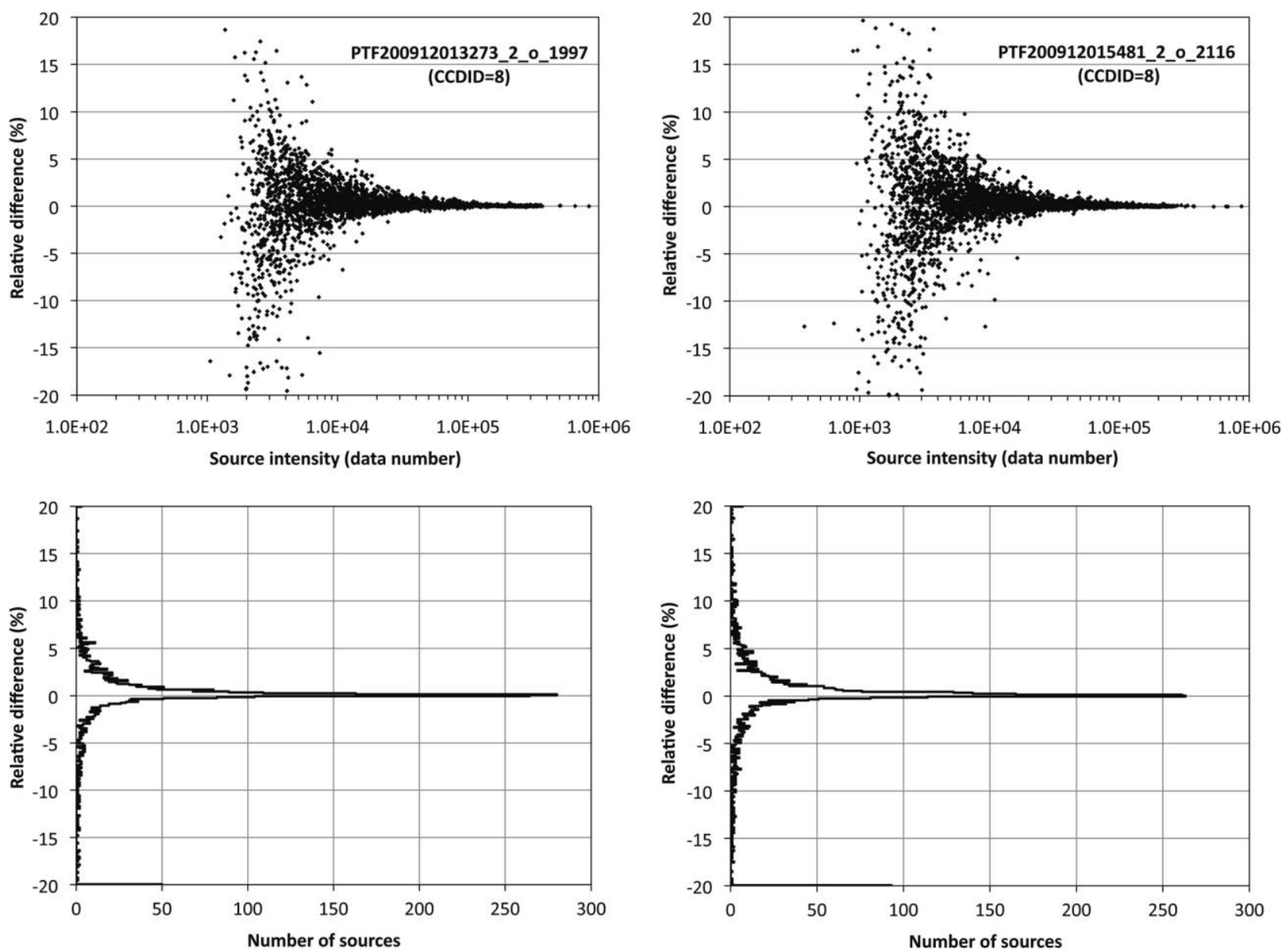

FIG. 5.-Comparison of source intensity computed by APT vs. SExtractor for PTF case 1 (left) and case 2 (right). Top: Scatter plots of the relative percentage differences in source intensities between SExtractor and APT as a function of source intensity for all filtered sources. Bottom: Histograms of the same relative percentage differences. The agreement between APT and SExtractor, as shown by these results, is excellent.

For both cases, the outer edge of APT's circular sky annulus is inscribed in the outer edge of SExtractor's square sky annulus, and the same for the inner edges. Thus, the SExtractor and APT settings are about as close as we can come to having equivalent sky annulus sizes between the two software programs while not having the inner dimension of the sky annulus smaller than the aperture (see earlier discussion in $\S 3.1$ ).

The number of sources obtained directly from SExtractor execution for cases $\mathrm{A} i$ and $\mathrm{B} i$ are listed in Table 4. Many more sources are seen at the shorter infrared wavelengths relative to the longer ones, as expected. We applied source filtering to the Spitzer IRAC sources, similar to that described above for PTF sources (including discarding sources with SExtractor FLAGS $>0$ ). This resulted in differing numbers of filtered sources between the two sky annulus cases (more on this in $\S 4.2)$.

The procedure that we followed for executing APT on the Spitzer IRAC mosaics is identical to that already described above for PTF images.

\subsection{Specifics for $H S T$ Image}

The HST image is packaged in a multiextension FITS (MEF) file, which contains a drizzle image, a weight image, and a context image. We split the MEF file into separate FITS files, each containing one image. We substituted NaN values into the drizzle image for pixels having zero weight. We also added the constant background value to the image data, which was previously removed by the HLA processing.

In order to minimize the effects of having multiple sources within an aperture, we set the aperture radius to 5 pixels (or $0.125^{\prime \prime}$ ) for the aperture photometry calculations. This is about half the size of the image's PSF, which has wings that extend a couple of pixels beyond the main lobe, and so an aperture correction would normally be required for absolute photometric calibration. Nevertheless, it yields integrated intensities that are within $\approx 5 \%$ of the total flux density of a moderately bright source. As before, since we are comparing APT versus SExtractor results, we can ignore the aperture correction for a basic comparison. 

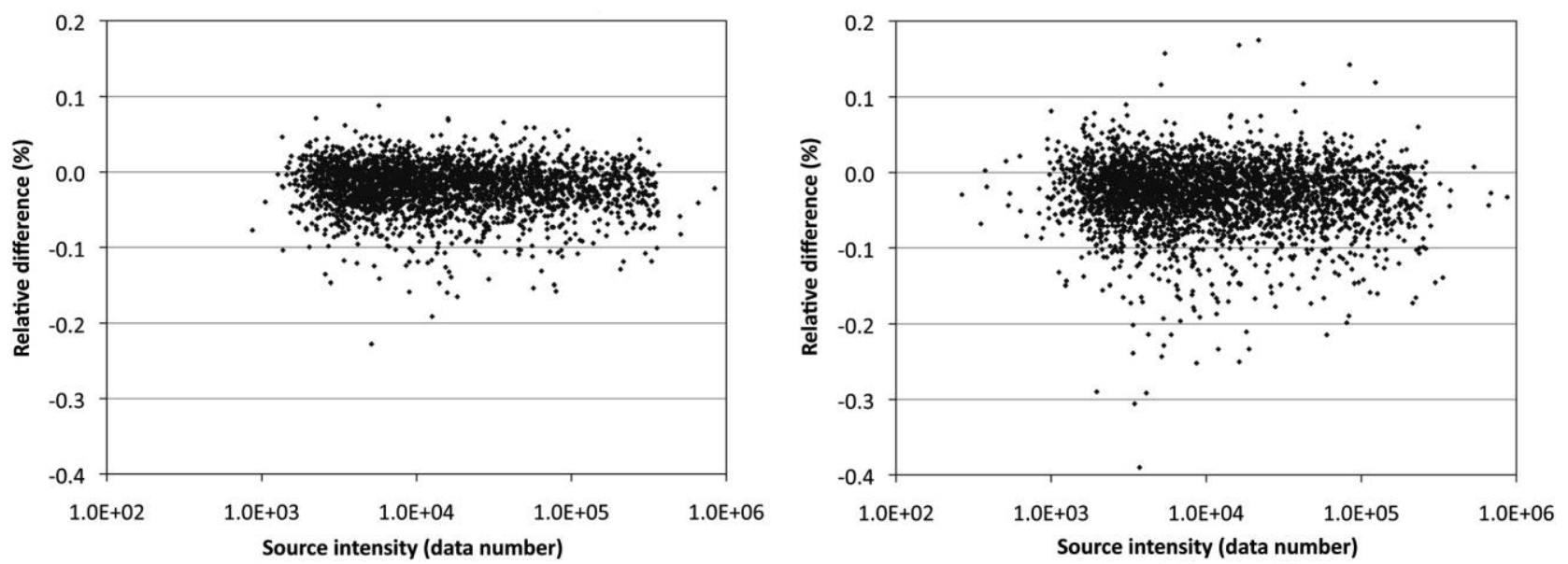

FIG. 6. - Comparison of sky background computed by APT vs. SExtractor for PTF case 1 (left) and case 2 (right). Shown are scatter plots of the relative percentage differences in sky background between SExtractor and APT as a function of source intensity for all filtered sources.

We chose a relatively small sky annulus for the background calculation, which seemed like a good choice for a moderately crowded field. We set BACK_SIZE $=40$ and BACKPHOTO_ THICK $=10$ pixels for SExtractor, and $r_{\text {in }}=10$ and $r_{\text {out }}=$ 20 pixels for APT. The outer edge of APT's circular sky annulus is inscribed in the outer edge of SExtractor's square sky annulus, which is similar for the inner edges. The inner radius was deliberately chosen to be substantially larger than the aperture radius to keep the annulus away from the PSF wings and to thus minimize the contribution by the source of interest to the computed background.

A total of 4901 sources were obtained directly from SExtractor execution. We applied source filtering to the HST sources, similar to that described for PTF sources in § 3.1. As part of the filtering that differed from the earlier description, we discarded the 31 sources that are within $0.2^{\prime \prime}$ (or 8 pixels) of another source. This left 962 sources for the SExtractor versus APT comparison. We note that doubling the angular separation allowed for nearest neighbors, with the aim of further reducing the crowding, would have decreased the number of sources available for our comparison by more than a factor of 2 .

The procedure that we followed for executing APT on the $H S T$ image is identical to that already described above for the PTF images.

\section{RESULTS AND DISCUSSION}

\subsection{PTF Image Comparison}

The results of our comparison of APT and SExtractor source intensities extracted from the PTF images are given in Table 3 and Figures 5-8. We used equation (1) to compute the relative percentage differences between source intensities from SExtractor and source intensities from APT, for all filtered sources from PTF cases 1 and 2. The agreement between APT and SExtractor, as shown by these results, is excellent. The median and modal values of $\Delta_{\text {rel }}^{i}$ for source intensity in Table 3 for each of the two cases is nearly zero, and the corresponding statistical dispersions of the data distributions are only $1-2 \%$. The top panels of Figure 5, which are plots of $\Delta_{\text {rel }}^{i}$ for source intensity as a function of source intensity, show that the $\Delta_{\text {rel }}^{i}$ values are large only for the faintest sources. The bottom panels of Figure 5 show data histograms that peak at nearly the same value, with the histogram for case 2 having extra data in its wings.

The data histograms have a small positive skewness (more so for case 2), which indicates SExtractor's tendency to slightly overestimate the source intensity relative to APT (or APT's tendency to slightly underestimate the source intensity relative to SExtractor). This is likely explained by the different methods of sky background estimation employed in SExtractor versus APT. Note that the nonzero histogram values at $-20 \%$ and $20 \%$ indicate that bins outside this range contain outlying data (which are not shown).

Upon examination of the results for a sky background subtraction effect on the source intensities in comparing the two cases of differing image-background levels, we were surprised to find that the statistical dispersion of $\Delta_{\text {rel }}^{i}$ for case 2 is actually about $30 \%$ larger than for case 1, as illustrated by Figure 6 , where we used an equation similar to equation (1) to compute the relative percentage differences in the sky backgrounds from SExtractor versus APT. However, the higher scatter for case 2 is due to the additional number of sources with intensities below about $5000 \mathrm{DN}$ that were extracted because of case 2's lower noise floor. When we include only sources above $5000 \mathrm{DN}$ in the statistical calculations, the dispersion for case 2 becomes about $15 \%$ smaller than for case 1 , which hints at the existence of the sky background subtraction effect we sought.

The plots in Figure 7 show intriguing comparisons of the source-intensity uncertainty computed by APT versus SExtractor for cases 1 (left) and 2 (right). Again, we used an equation similar to equation (1) to compute the relative percentage 

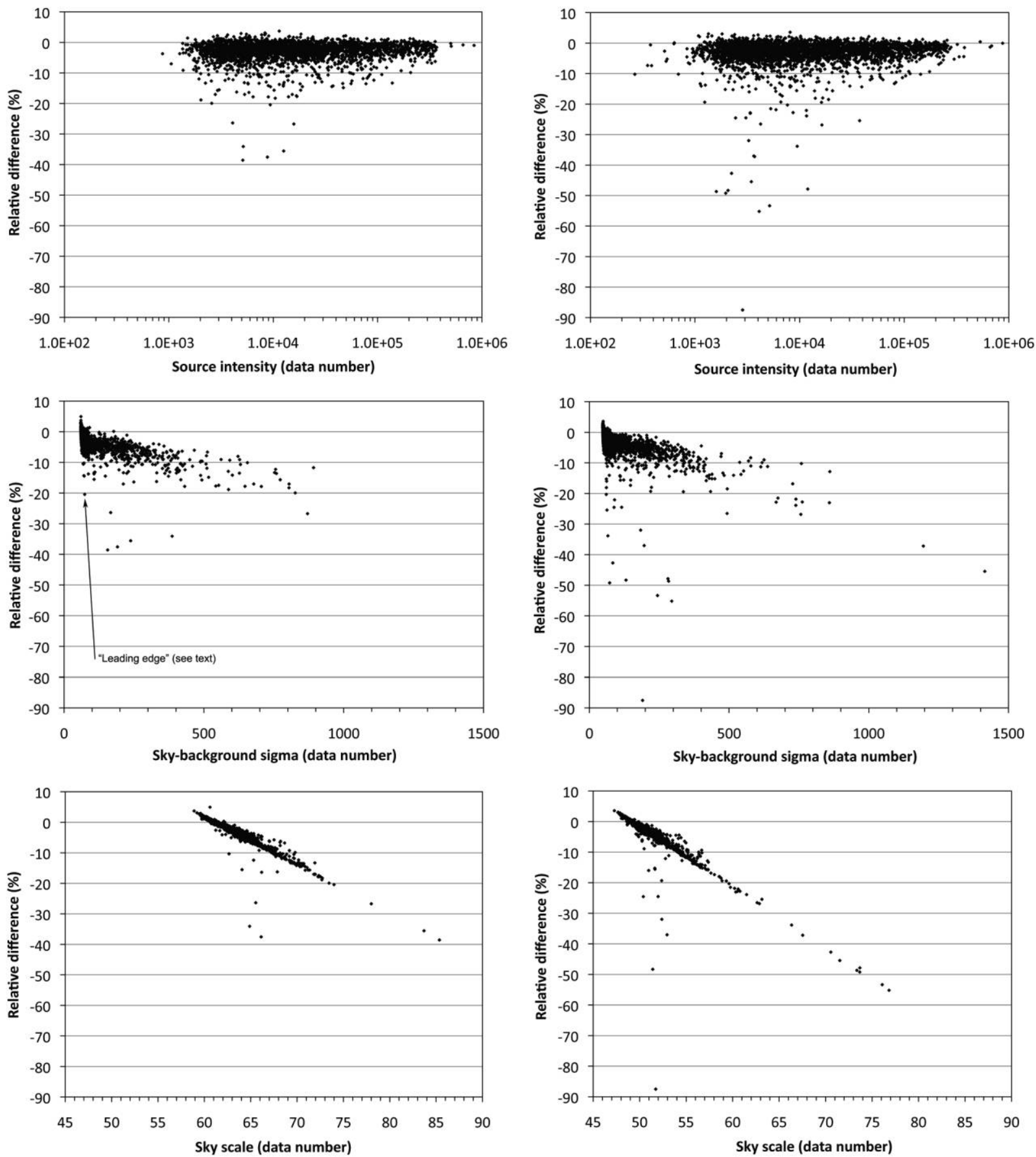

FIG. 7.-Comparison of source-intensity uncertainties computed by APT vs. SExtractor for PTF case 1 (left) and case 2 (right). Top: Scatter plots of $\Delta_{\text {rel }}^{i}$ for sourceintensity uncertainty as a function of source intensity. Middle: Scatter plots of the same relative percentage differences as a function of sky background sigma. Bottom: Panels are scatter plots of the same relative percentage differences as a function of sky scale.

differences in the source-intensity uncertainties from SExtractor versus APT. Data points for all of the filtered sources of cases 1 and 2 are shown. The top panels are scatter plots of $\Delta_{\text {rel }}^{i}$ for source-intensity uncertainty as a function of source intensity, which have median values of $-2.49 \%$ for case 1 and $-2.37 \%$ for case 2 (these values both have a statistical dispersion of approximately $2.3 \%$ ). As might be expected, the larger discrepancy occurs for case 1, which has the higher sky background of the two cases. The negative values indicate that APT overestimates the source-intensity uncertainty relative to SExtractor, which may not be a surprising result, because APT includes the uncertainty of the sky background estimation, whereas 

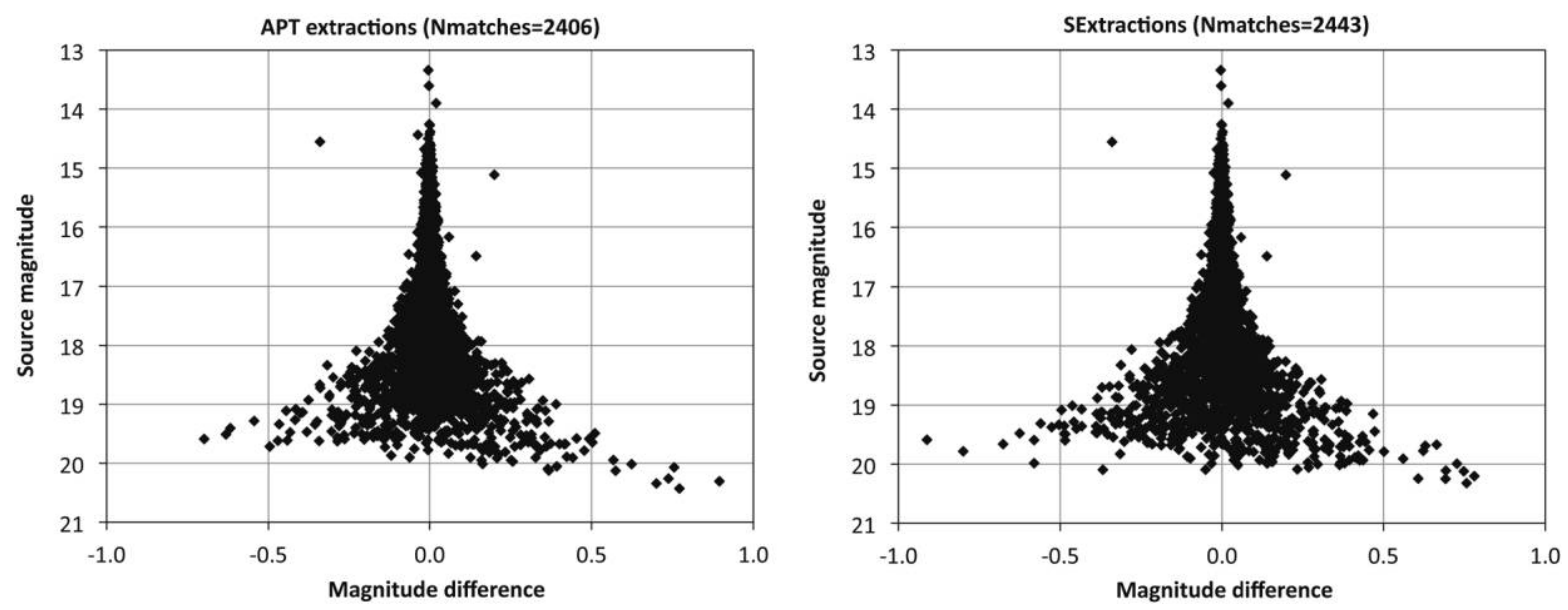

FIG. 8. - Plots of source magnitude of case 1 vs. source magnitude of case 1 minus source magnitude of case 2 for source matches between the two PTF cases, as computed by APT (left) and SExtractor (right).

SExtractor does not, according to Bertin (2006). Moreover, a comparable plot of SExtractor's FLUXERR (source-intensity uncertainty) versus FLUX (source intensity) gives a locus of data points that smoothly follow the lower edge of the scattered APT data points, which is consistent with the statement that SExtractor's estimate of the source-intensity uncertainty can only be regarded as a lower limit (Bertin 2006). Our crude simulations of folding the uncertainty of the sky background estimation into SExtractor's source-intensity uncertainties improve the aforementioned median values of $\Delta_{\text {rel }}^{i}$ for sourceintensity uncertainty to about $-1 \%$ for both cases.

The middle panels in Figure 7 are scatter plots of $\Delta_{\text {rel }}^{i}$ for source-intensity uncertainty as a function of sky background sigma. One can start to see a dependence of $\Delta_{\text {rel }}^{i}$ for sourceintensity uncertainty on sky background sigma. This is even more apparent in the bottom panels, which are scatter plots of $\Delta_{\text {rel }}^{i}$ for source-intensity uncertainty as a function of sky scale that show a superb linear relationship. Comparing the middle and bottom panels demonstrates how the dependence of $\Delta_{\text {rel }}^{i}$ for source-intensity uncertainty on sky scale has mostly "collapsed" horizontally onto a line that coincides with the obvious lowerrange leading edge of the data points in the graph of $\Delta_{\text {rel }}^{i}$ for source-intensity uncertainty on sky background sigma (the line formed by the data in the bottom panel is the same as the lowerrange leading edge of the data in the corresponding middle panel, which is not readily apparent because the horizontal axes have different scales). Thus, it can now be easily seen that the discrepancy between source-intensity uncertainties from APT versus SExtractor increases with increasing sky scale. It is unclear whether this effect can be attributed to the uncertainty in sky background estimation, as opposed to the sky background estimation itself. Further investigation is beyond the scope of this article.

As a final figure of merit, we studied the variation of source intensities between the two PTF exposures. We matched sources from the APT results between cases 1 and 2 and obtained 2406 matches using a search radius of $1^{\prime \prime}$. We did the same for the SExtractor results and got 2443 matches. Presumably, the number of APT matches is different from the number of SExtractor matches because of small differences in how the two software programs compute the centroid position of the sources, and evidence for this is that sometimes more matches are yielded for APT than for SExtractor and vice versa, depending on the data. We converted PTF source intensities into magnitudes by dividing them by the exposure time (the same for both exposures) and applying the magnitude zero points listed in Table 1 in the usual way. We made plots of source magnitude of case 1 versus source magnitude of case 1 minus source magnitude of case 2 for source matches between the two PTF cases. Figure 8 shows the separate graphs for results from APT (left) and SExtractor $(r i g h t)$. Both graphs look very similar, and the median of the magnitude differences for each graph is less than $1 \mathrm{mmag}$.

\subsection{Spitzer IRAC Mosaic Comparison}

The results of our comparison of SExtractor and APT source intensities extracted from the Spitzer IRAC mosaics are given in Table 4 and Figures 9-12. The agreement is generally very good, although not as good as for the PTF images (see $\S 4.1$ ). Many of the features already discussed for the PTF images are present in these comparisons and will not be repeated. There are interesting new ones, as well, and these are discussed as follows.

The noise in the Spitzer IRAC data is higher for channels 3 and 4 relative to channels 1 and 2 (according to the Spitzer IRAC instrument handbook, the mean number of noise pixels is $7.0,7.2,10.8$, and 13.4 for channels $1-4$, respectively), and the threshold for source detection is sensitive to the noise. Hence, the results for Spitzer IRAC channels 1 and 2 have many more low-intensity sources with high signal-to-noise ratios than the results for channels 3 and 4 (see Table 4). The number of 

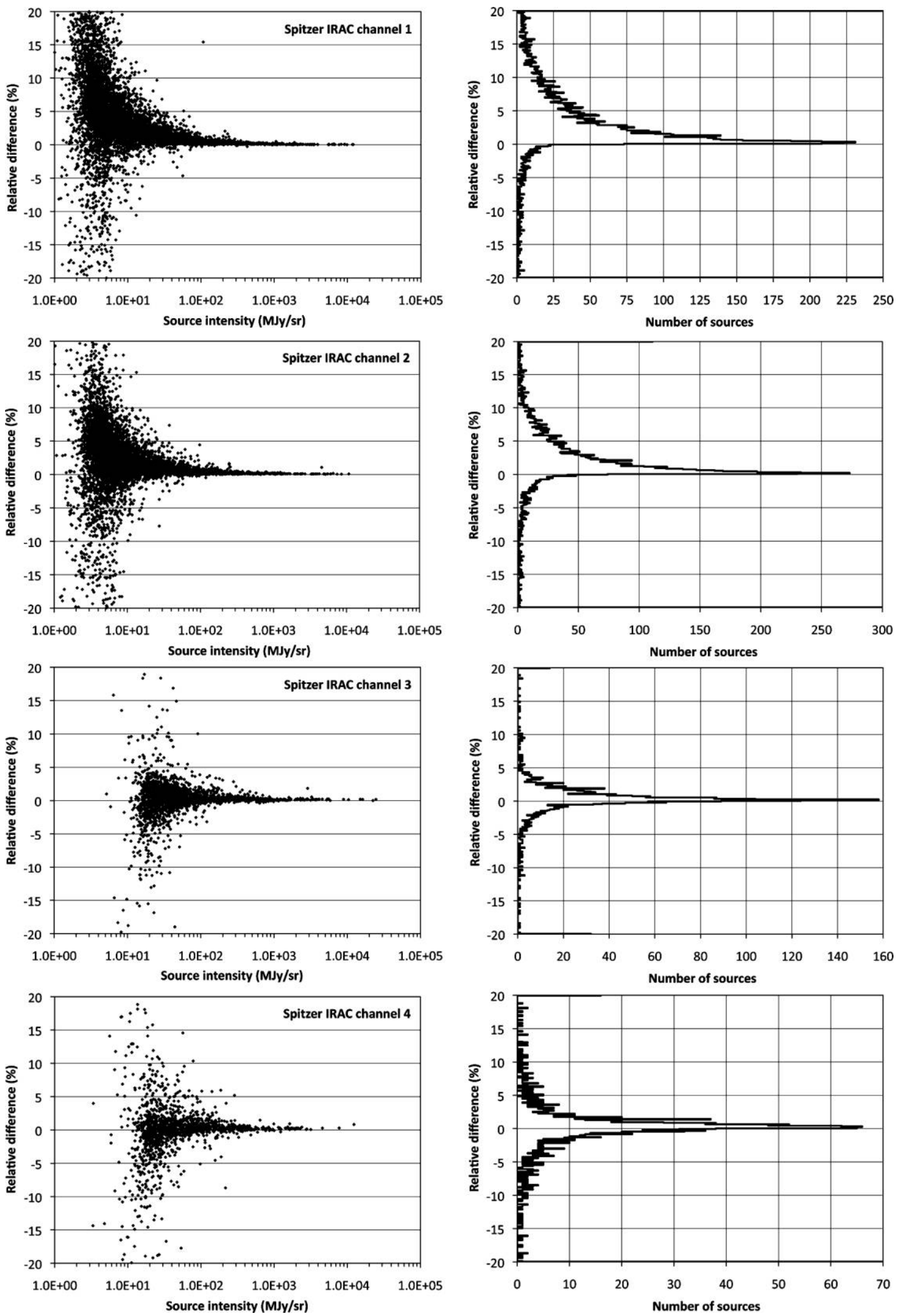

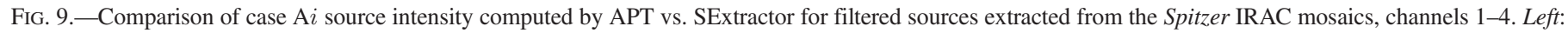

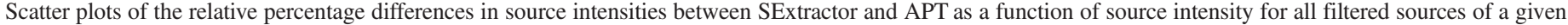
Spitzer IRAC channel. Right: Each panel corresponds to the adjacent left panel and is a histogram of the same relative percentage differences. 

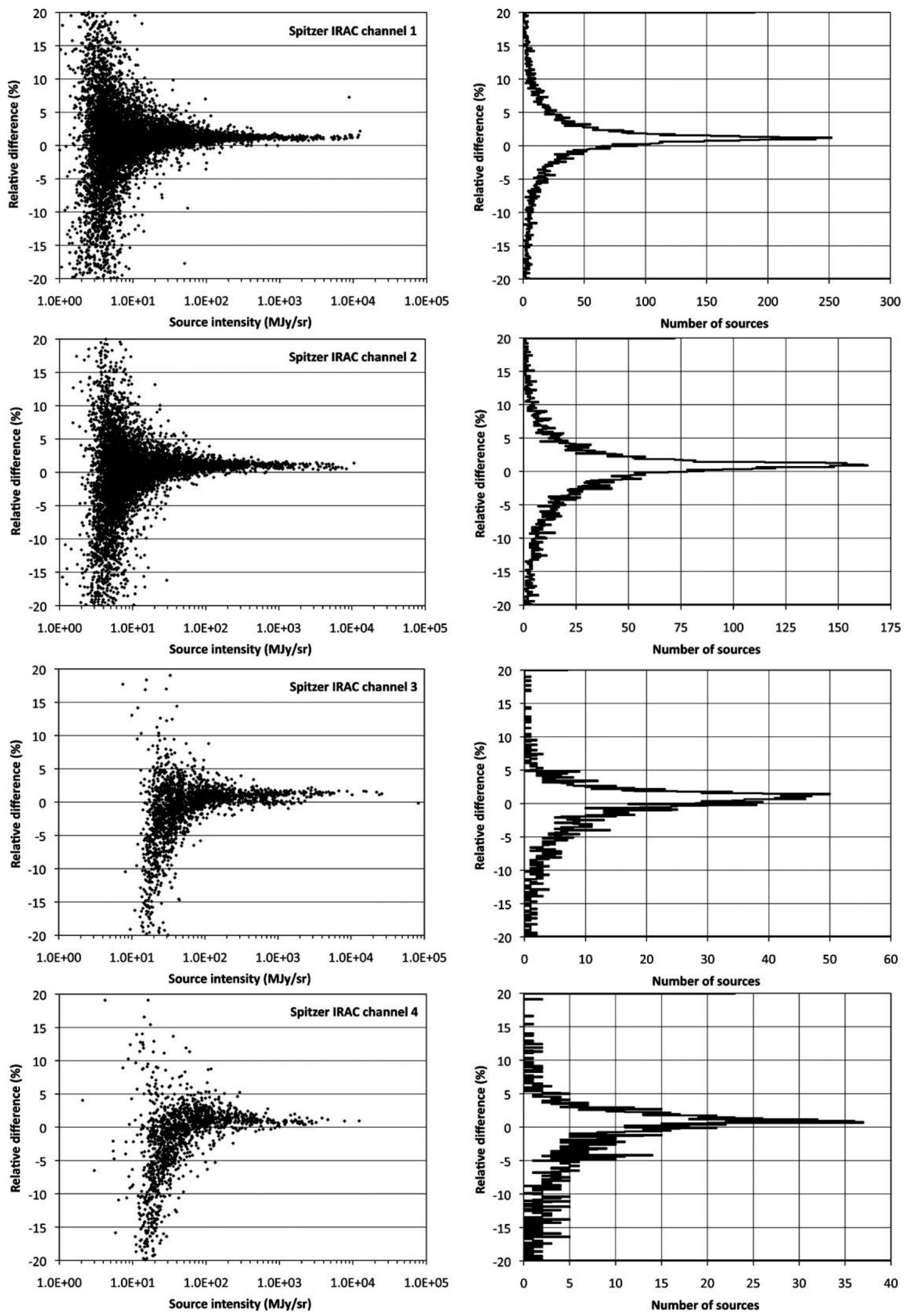

FIG. 10.-Comparison of case B $i$ source intensity computed by APT vs. SExtractor for filtered sources extracted from the Spitzer IRAC mosaics, channels 1-4. Left: Scatter plots of the relative percentage differences in source intensities between SExtractor and APT as a function of source intensity for all filtered sources of a given Spitzer IRAC channel. Right: Each panel corresponds to the adjacent left panel and is a histogram of the same relative percentage differences. 

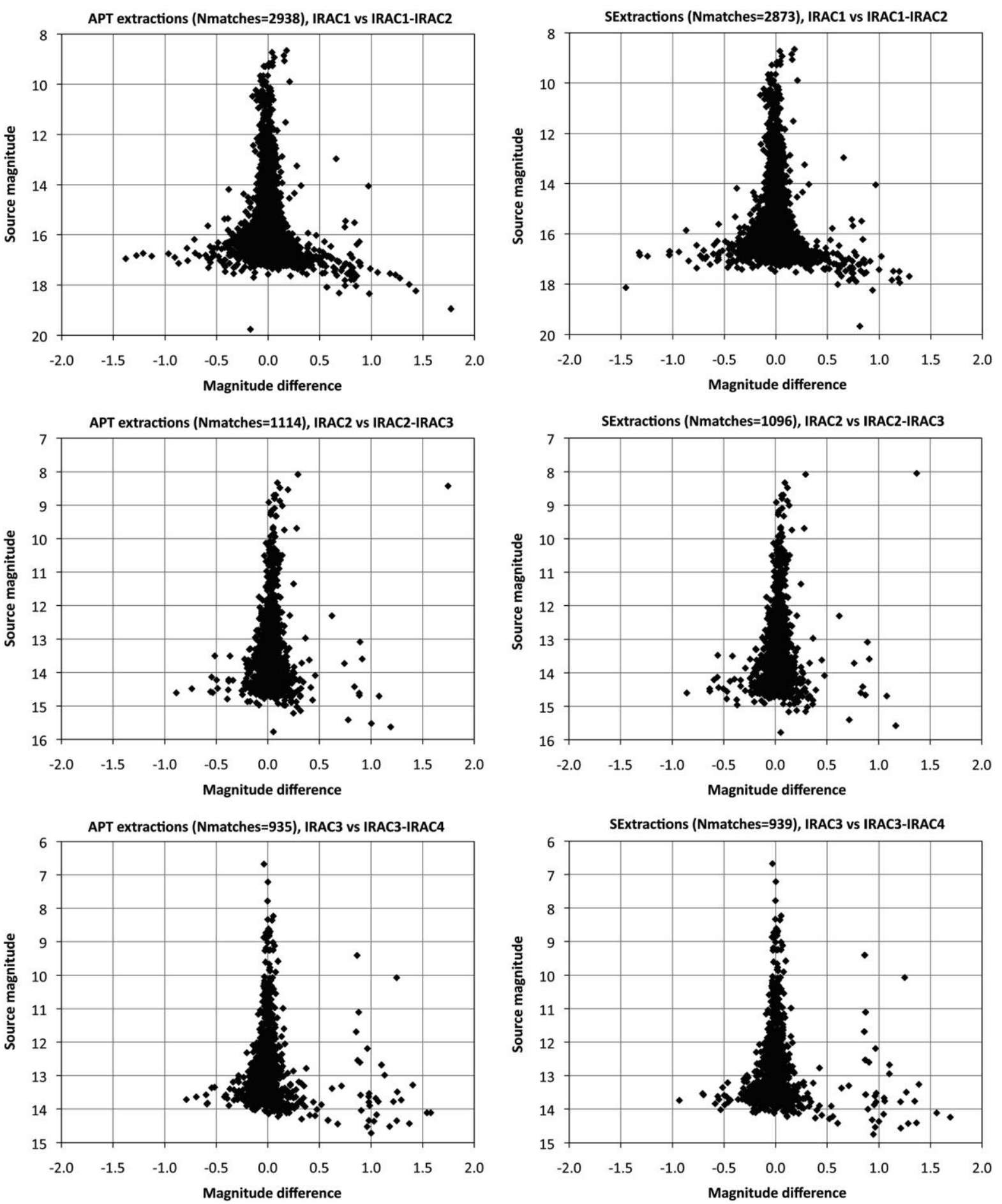

FIG. 11.-Plots of source magnitude vs. magnitude difference between adjacent Spitzer IRAC channels (see graph titles) for cross-channel source matches from cases $\mathrm{A} i$, as computed by APT (left) and SExtractor (right). See text for further details.

extracted sources varies somewhat between the cases for large versus small sky annuli, which is also due to source detections being thresholded relative to the variability of the sky background, which in turn depends on the sky annulus size (because
BACK_SIZE and BACKPHOTO_THICK differ between the two scenarios).

Curiously, the lower-channel histograms for the large sky annulus cases shown in Figure 9 have positive skewness, which 

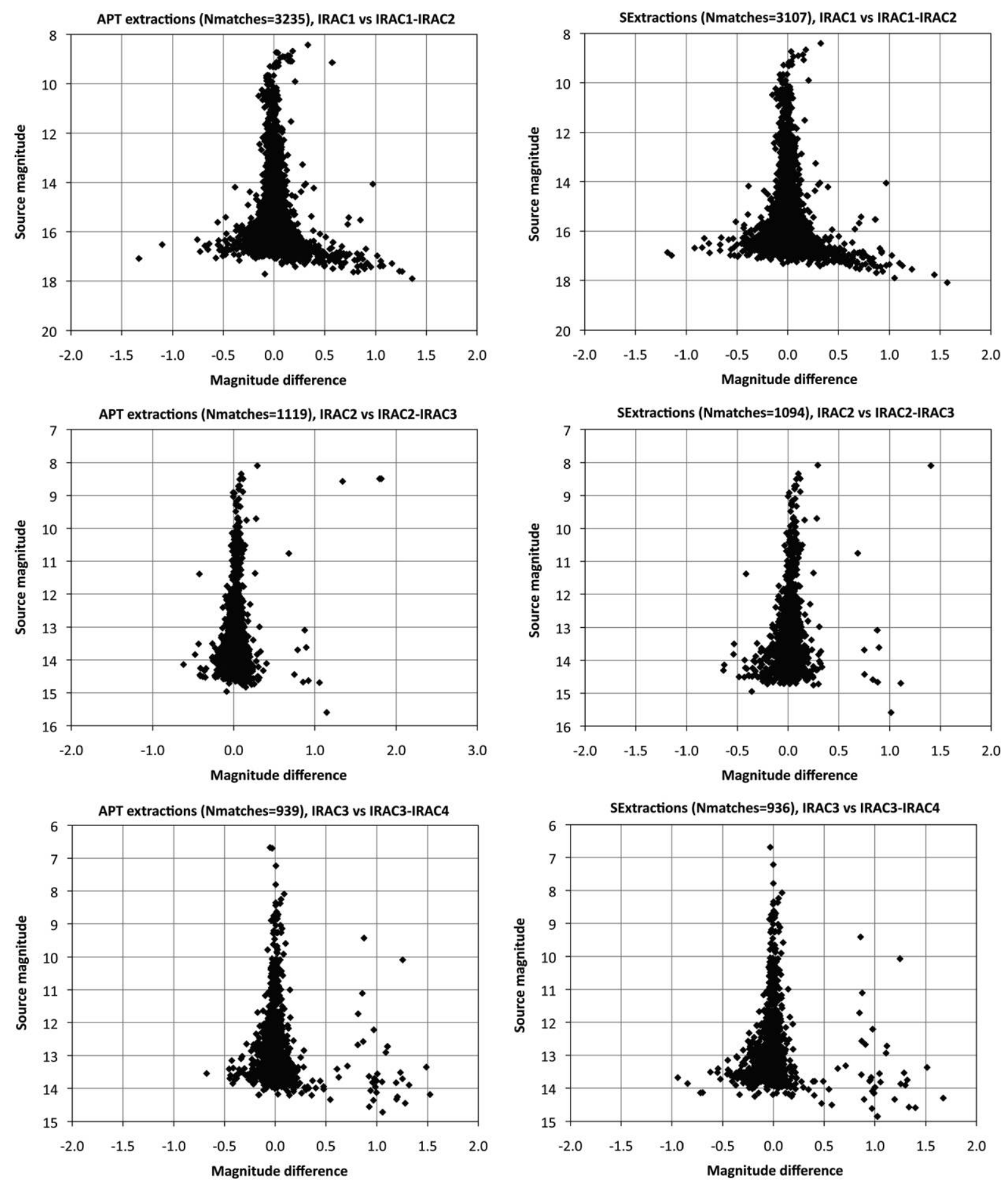

FIG. 12.-Plots of source magnitude vs. magnitude difference between adjacent Spitzer IRAC channels (see graph titles) for cross-channel source matches from cases $\mathrm{B} i$, as computed by APT (left) and SExtractor (right). See text for further details.

displaces the medians from the modes of the distributions of $\Delta_{\text {rel }}^{i}$ for source intensities. The large sky annulus cases have $\Delta_{\text {rel }}^{i}$-distribution modes for source intensity near $0 \%$ (see Table 4), but the positive skewness present for channels 1 and 2 causes the median values of $\Delta_{\text {rel }}^{i}$ for source intensity to deviate to $+2.00 \%$ and $+1.20 \%$, respectively. Figure 10 presents graphs of the small sky annulus results and shows that the histograms for channels 1 and 2 have very little skewness and the histograms for channels 3 and 4 have negative skewness. The small sky annulus cases have $\Delta_{\mathrm{rel}}^{i}$-distribution modes for 

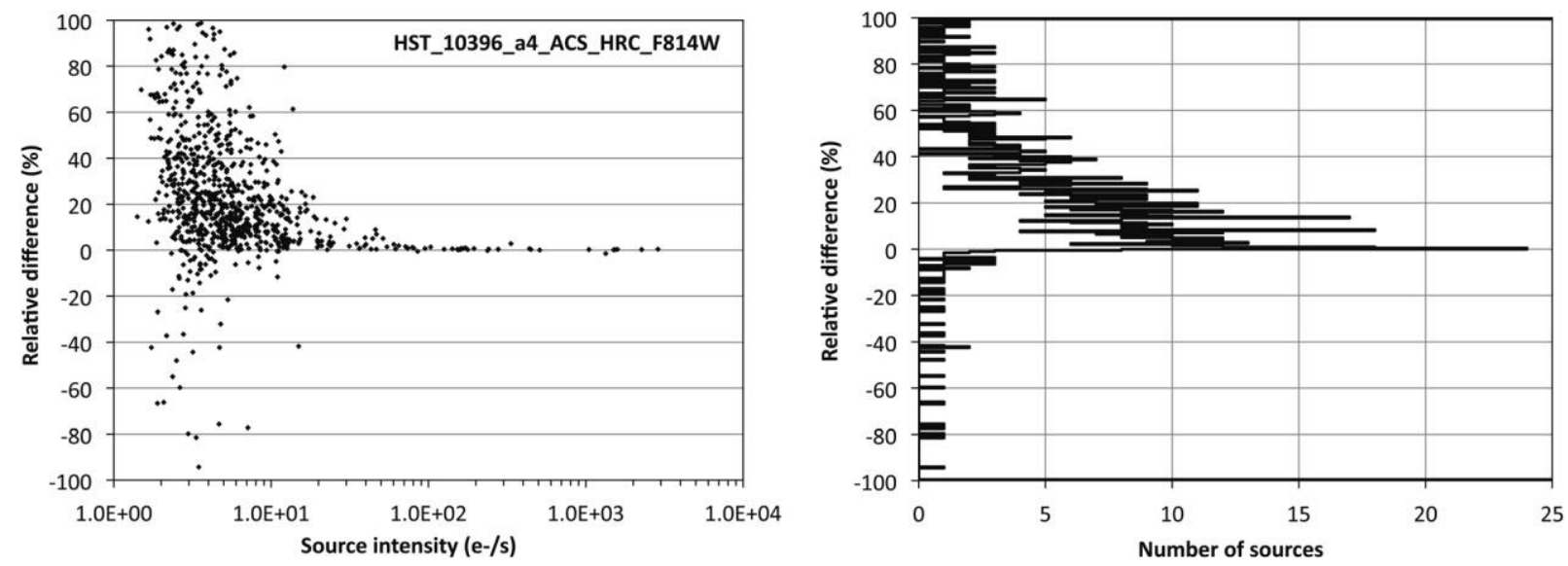

FIG. 13.-Comparison of source intensity computed by APT vs. SExtractor for the HST image case. Left: Scatter plot of the relative percentage differences in source intensities between SExtractor and APT as a function of source intensity for all filtered sources. Right: Histogram of the same relative percentage differences. The agreement between APT and SExtractor, as shown by these results, is excellent for relatively bright sources.

source intensity hovering around $1 \%$, but the negative skewness in the distribution causes the median values of $\Delta_{\text {rel }}^{i}$ for source intensity to progressively range from $+0.92 \%$ for channel 1 to $-0.47 \%$ for channel 4 .

The bias and skewness in the data histograms indicate a tendency in one of the software programs to overestimate or underestimate the source intensity relative to the other software program (depending on the sign of the skewness). The only difference in inputs between cases $\mathrm{A} i$ and cases $\mathrm{B} i$ for a given instrument channel $i$ is the size of the sky annulus. Therefore, we can assume that the differences between the two sets of cases for APT and SExtractor results arise from the different sky annulus geometries and sizes and the different methods employed to compute the sky background between the two software programs. The small sky annulus cases have $\Delta_{\mathrm{rel}}^{i}$-distribution modes for source intensity around $1 \%$, which may be caused by SExtractor's square sky annulus relative to APT's circular sky annulus, an effect that would be expected to be more pronounced for the small sky annulus cases than for the large. Another possibility is the differences in how the background is computed between APT and SExtractor. While APT does 3- $\sigma$ data clipping in a single pass and then computes the median as an estimate of the background (for the results herein), SExtractor's background estimation involves iterative clipping until convergence at 3- $\sigma$ and then computing either the mean, if $\sigma$ has changed by less than $20 \%$, or computing the mode, where the mode is given via the empirical formula

$$
\text { mode }=(2.5 \times \text { median })-(1.5 \times \text { mean })
$$

(Bertin \& Arnouts 1996). In all cases, the data-distribution dispersions are 2-6 times larger than the corresponding median and modal values.

We also studied the variation of source intensities of the same astronomical objects identified in adjacent IRAC channels, and this involved plotting their magnitudes in the shorter infrared channel versus color (magnitude differences) between the two channels of interest (see Figs. 11 and 12). Generally, if the photometry is done correctly, we expect the color of most of the sources (field stars) to be near zero, with more scatter for the fainter sources. Using a search radius of $1^{\prime \prime}$, we matched sources from the APT results between IRAC channels 1 and 2 . We also performed the matching between channels 2 and 3 and between channels 3 and 4 . We did all of this separately for cases $\mathrm{A} i$ and $\mathrm{B} i$. We then repeated this process for the SExtractor results. In most of the cases, there were more matches found in the APT results than in the SExtractor results, although one must be careful to not read too much into this (see the discussion at the end of $\S 4.1$ ). Using the channel-dependent aperture corrections and magnitude zero points listed in Table 2, we converted Spitzer source intensities into magnitudes and computed the aforementioned magnitude differences. For cases $\mathrm{A} i$ and $\mathrm{B} i$, respectively, Figures 11 and 12 show separate graphs of results from APT (left) and SExtractor (right) with side-by-side comparisons. Each graph of the APT results is quantitatively very similar to the corresponding graph of the SExtractor results, and the absolute differences between their median magnitude differences are within approximately $1 \mathrm{mmag}$ for the large sky annulus cases and $10 \mathrm{mmag}$ for the small sky annulus cases.

We do not include a discussion herein of the source-intensity uncertainties computed by APT and SExtractor for the Spitzer IRAC mosaics. This is because, for sources extracted from mosaics, the source-intensity uncertainty also depends on the mosaic's depth of coverage, as well as pixel-to-pixel correlations, and neither APT nor SExtractor properly account for these effects (although SExtractor can account for the former via an input "weight" map). Version 1.0.5 of APT has a single depth-of-coverage input parameter, which may be useful for bracketing the source-intensity uncertainty of a given source. The Spitzer mosaics studied here have depth of coverage that 
(STScI/NASA), the Space Telescope European Coordinating Facility (ST-ECF/ESA), and the Canadian Astronomy Data Centre (CADC/NRC/CSA). This research made use of Montage, funded by NASA's Earth Science Technology Office,
Computation Technologies Project, under cooperative agreement NCC5-626 between NASA and the California Institute of Technology. Montage is maintained by the NASA/IPAC Infrared Science Archive.

\section{REFERENCES}

Bertin, E. 2006, SExtractor, Version 2.5, User's Manual (Paris: IAP) $\rightarrow$ Bertin, E., \& Arnouts, S. 1996, A\&AS. 117, 393

$\rightarrow$ Fazio, G. G., Hora, J. L., Allen, L. E., Ashby, M. L. N., Barmby, P., Deutsch, L. K., Huang, J.-S., Kleiner, S., et al. 2004, ApJS, 154, 10

Fiorentino, G., Contreras, R., Clementini, G., Glatt, K., Sabbi, E., Sirianni, M., Grebel, E., \& Ghallager, J. 2008, Mem. Soc. Astron. Italiana, 79, 3

Grillmair, C. J., Laher, R., Surace, J., Mattingly, S., Hacopians, E., Jackson, E., van Eyken, J., McCollum, B., et al. 2010, in ASP Conf. Ser. 434, (San Francisco: ASP), 28

Holwerda, B. W. 2005, Source Extractor for Dummies (5th ed.; Baltimore: STScI)

Koekemoer, Anton M., Fruchter, Andrew S., Hook, Richard, \& Hack, Warren 2002, in 2002 HST Calibration Workshop (Baltimore: STScI), 337 $\rightarrow$ Laher, R. R., Gorjian, V., Rebull, L. M., Masci, F. J., Fowler, J. W., Helou, G., Kulkarni, S. R., \& Law, N. M. 2012, PASP, 124, 737

$\rightarrow$ Law, N. M., Kulkarni, S. R., Dekany, R. G., Ofek, E. O., Quimby, R. M., Nugent, P. E., Surace, J., Grillmair, C. J., et al. 2009, PASP, 121,1395

$\rightarrow$ Makovoz, D., \& Marleau, F. R. 2005, PASP, 117, 1113

$\rightarrow$ Rau, A., Kulkarni, S., Law, N. M., Bloom, J. S., Ciardi, D., Djorgovski, G. S., Fox, D. B., Gal-Yam, A., et al. 2009, PASP, 121, 1334

Rebull, L. M., Gorjian, V., Squires, G., \& The NITARP Team 2011a, BAAS, 43, 248.11

$\rightarrow$ Rebull, L. M., Johnson, C. H., Hoette, V., Kim, J. S., Laine, S., Foster, M., Laher, R., et al. 2011b, AJ, 142, 25

$\rightarrow$ Werner, M. W., Roellig, T. L., Low, F. J., Rieke, G. H., Rieke, M., Hoffman, W. F., Young, E., Houck, J. R., et al. 2004, ApJS, 154, 1 